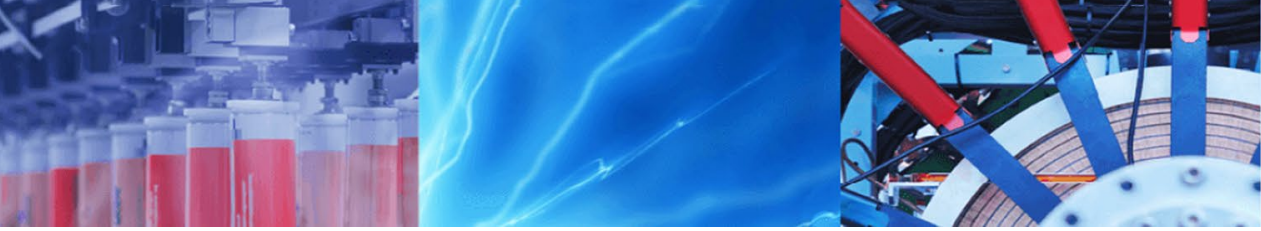

Research Article

\title{
Mathematical modeling and experimental evaluation of a prototype double-tube Magnetorheological damper
}

\author{
Ebrahim Yarali ${ }^{1}$ - Arman Mohammadi ${ }^{1} \cdot$ Sheyda Mafakheri ${ }^{2} \cdot$ Mostafa Baghani $^{1} \cdot$ Hamed Adibi $^{3}$
}

(c) Springer Nature Switzerland AG 2019

\begin{abstract}
The performance of Magnetorheological dampers should be analyzed to study the stability of Magnetorheological fluid and its configuration. To this end, in this study a prototype Magnetorheological fluid is presented. The fluid consists of stabilized Silicone dioxide $\left(\mathrm{SiO}_{2}\right)$ nanoparticles, Stearic acid, Phosphoric acid and micron-sized soft ferromagnetic carbonyl iron particles. To assure the authentic performance, sedimentation and magneto-rheometry tests are conducted. Also, a prototype of double-tube Magnetorheological damper with double magnetic components is fabricated by using the mentioned magnetorheological fluid characteristics. Damping force measurement test is carried out to measure the damping force in various electrical currents. The Kwok model is employed to examine the analytical model predictions against the experiments. Furthermore, a general evolution is presented using the neural network algorithm. It is demonstrated that the damping force in saturated current is almost five times higher than in the zero current. In addition, the derived evolution for the damping force has a high performance to predict the response of the Magnetorheological damper in other electrical currents.
\end{abstract}

Keywords Magnetorheological fluid · Magnetorheological damper · Carbonyl iron particle · Silicone dioxide nanoparticles $\cdot$ Neural network algorithm $\cdot$ Mathematical modeling

\section{Introduction}

Some properties of smart materials change dramatically in response to external stimuli, such as temperature, $\mathrm{pH}$, magnetic and electric field, chemical compounds, and light [1-4]. Smart fluid such as Magnetorheological, Electrorheological, Ferro and Ferro based-on-magnetorheological fluids, are an extensive class of smart materials, despite of shape memory polymer (SMP), shape memory alloy, Hydrogel, Magnetorheological elastomer and etc. [5, 6]. Electrorheological fluids (ER fluids) were first discovered in 1949 by Winslow [7]. ER fluids are excited by an electric field (as an external stimulus); also, some changes in their apparent viscosity can significantly affect their rheological properties. It is well known that MR fluid is a group of smart fluids, which alter their rheological properties in response to a magnetic field as external stimuli [8-10]. MR fluid initially discovered by Rabinow at the US National Bureau of Standards in 1948 [11]. MR fluids have lower yield shear strength, lower-temperature range, and higher energy consumption compared to their counterparts. Regarding these characteristics, MR fluid is replaced with ER fluid after almost one decade from discovering of ER fluids [12-14]. Ferro fluids, discovered in 1960s, are actually a colloidal suspension of nanoparticle distributed uniformly in the carrier fluid (based fluid) and surrounded by a special surfactant. These fluids do not settle easily in presence of the magnetic field, gravity field, and centrifugal forces center. Ferro-fluids have much lower yield stress compared to other fluids, as well as their high stabilization. Therefore, the combination

\footnotetext{
$\triangle$ Mostafa Baghani, baghani@ut.ac.ir|'School of Mechanical Engineering, College of Engineering, University of Tehran, Tehran, Iran. ${ }^{2}$ Department of Civil Engineering, University of Kurdistan, Kurdistan, Iran. ${ }^{3}$ Department of Mechanical Engineering, Amirkabir University of Technology, Tehran, Iran.
} 
of MR and Ferro-fluids called Ferro-fluid based-on MR fluids $[15,16]$. As mentioned, MR fluid is one of the most common smart fluids used as the suspension for micron sized magnetic particles dispersed in a non-magnetic liquid for nonNewton fluids. When these fluids are subjected to magnetic field, the magnetic particles are polarized. This makes particles orientate in parallel to the magnetic field and construct some chains of particles. Consequently, this effect makes them more viscous $[7,17]$. These super characteristics of MR fluids, make them potential candidates to be used in numerous applications, e.g. automotive industry, machining, military, medicine and aerospace devices such as shock absorber, clutch, brake, and suspension seat [7, 12, 18-21].

In devices with passive system control, the damping factor of the shock absorber is commonly constant; on the other hand, they have passive suspension system (because the type of hydraulic oil and orifice size during the operation are constant). Thus, for instance the passenger comfort and handling is not fully taken into account. Furthermore, in order to overcome these limitations, an active control system should be used. However, this system may require a high power supply as well as some different control loop elements such as sensors. Semi-active control systems often combine useful characteristics of these two types of controlling systems. For this purpose, usually, MR dampers are employed instead of active force generator elements $[22,23]$. In MR damper, the damping factor is changed due to the applied magnetic field. It alters the viscosity of MR fluid, which brings more comfort and convenience for the passenger [7, 24, 25]. MR dampers have many advantages such as large scope continual adjustable damping force, low consumption, simple structure, short time response and long-range controllable damping force $[26,27]$. The controllable MR based-on-MR fluid devices usually have five performance modes: valve mode, shear mode, squeeze mode, a combination of these three modes and helical mode. Between these modes, the squeeze mode is rarely used because its application is limited to low and limited amplitudes [28-31].

Until recently, several experiments and simulations have been conducted on MR dampers. In most of these cases, commercial MR fluid and MR damper have been used, while only a few studies focused on both designing and modeling of MR damper and its MR fluid. There are lots of researches in the literature on the modeling and testing various types of MR dampers. Normally a commercial MR damper or a prototype MR damper is tested and then employing a hysteresis model, the dynamic behavior of MR damper had been described [24, 32-34]. Chooi et al. [35] using analytical techniques proposed a general expression on the annular flow of fluids and comparing the results with those of experiments, they validated their formulation. Yu et al. [36] presented a new working mode for MR damper (named Helical mode) and studied the damping force of the MR damper. To arrive at a shorter response time, Lee et al. [37] experimentally investigated the response time of MR damper. In addition, other researchers [38-41] worked on the response time of MR damper. Some authors such as Peng et al. [42] reported a modeling and parametric study on MR dampers. Furthermore some authors considered the effect of temperature on performance and mathematical modeling of MR dampers [43].

In this study, in order to investigate the performance of a prototype MR damper, a prototype MR fluid is fabricated. Then, a configuration of the control valve with one conventional piston, two coils (as piston) and double-tube MR damper are designed and manufactured. A magnetic analysis is carried out to investigate the distribution of the magnetic flux in the MR fluid. Finally, to predict the dynamic behavior of the MR damper, a phenomenological model is utilized, where its parameters are identified through Genetic algorithm (GA). In the last section using the neural network, a general relation for the damping force incorporated to the magnetic field, frequency and the amplitude of rod piston is represented. We finally present a summary and give some concluding remarks.

\section{Theoretical foundations}

To design a Magnetorheological damper, it is important to characterize the MR fluid properties used in MR damper (e.g. yield stress, plastic viscosity). Different operation models can be used to properly model the mechanical behavior of MR fluid; Bingham plastic (BP), Herschel-Bulkley, and Bi-Viscous Model are some well-known models [28]. BP model is the usual one to analyze an MR fluid [44], so the BP model is utilized in this study, which could be expressed as $\tau=\tau_{y}(H) \operatorname{sgn}(\dot{\gamma})+\eta \dot{\gamma}[45,46]$ where $\tau$ is the shear stress of MR fluid, $\tau_{y}(H)$ is yield shear stress in the presence of magnetic field $H$ that indicates the strength of MRF under applying the magnetic field. $\dot{\gamma}$ stands for the strain rate applied to the MR fluid, $\eta$ denotes the plastic viscosity in the absence of magnetic field, and "sgn" is the signum function. As mentioned, MR devices operate in different operation modes such as valve mode, shear mode, helical mode, pressure mode and a combination of these modes. In this study, the operation mode for the MR damper is the combination of shear and valve modes. To derive equations of generated forces and dynamic domain of the MR damper, a combination of the Navier-Stokes, continuity and BP model are used as pointed out below, [47]:

$F=\left(\frac{12 \eta L_{a} A^{2}}{\pi h^{3}\left(h+D_{c}\right)}\right) v+\left(\frac{3 L_{a} \tau_{y}}{h}\right) A ; \quad D=\frac{\pi\left(h+D_{c}\right) \tau_{y} h^{3}}{4 \eta A v}$ 
in which $F$ and $D$ stand for the total generated damping force and the dynamic domain, which are equal to ratio of the generated total damping force to the non-controlled force, respectively. First term in Eq. (1) is induced by viscous component and the second term stands for the induced stress due to the magnetic field. Parameters $L_{a}$, $A_{p}, h, D_{c}$ and $v_{p}$ denote the pole length of coil, effective cross section of the control valve (piston), the thickness of the MR fluid between the inner cylinder and control valve (also called gap), inner diameter of the inner cylinder and velocity of piston rod, respectively. The schematic cross section of the control valve and its associated parameters are shown in Fig. 1.

Designing control valve (piston) of the MR damper is the next major step in the manufacturing of MR damper. Design parameters are the value of gap $(h)$, number of turns in the coil $\left(N_{c o}\right)$, diameter of the coil wire $\left(d_{w}\right)$, length of the wire wound $(L)$ and diameter of the piston $\operatorname{rod}\left(d_{\text {rod }}\right)$. First, considering the maximum force of the target design, the diameter of the piston rod according to the yield stress of the piston rod is computed as $d_{\text {rod }}=2 \sqrt{F_{\text {Max }}} / \pi \sigma_{y}$ where $\sigma_{y}$ is the yield stress of the piston and $F_{\max }$ is the maximum applied force to the piston. Also, diameter of the coil wire is calculated from the electric current density as $d_{w}=0.255 /[48,49]$, where $I$ is the maximum current at which the MR fluid is saturated. The saturated current is found through performing a magneto-rheometry test on the MR fluid. In addition, to determine the number of coil wound, by applying the Ampere law to the control valve, we have $N_{c o}=2 H_{m r} h / I[50,51] . H_{m r}$ is the intensity of the magnetic field at which MR fluid is saturated. The other geometrical parameters of the coil core are calculated as below [50]:

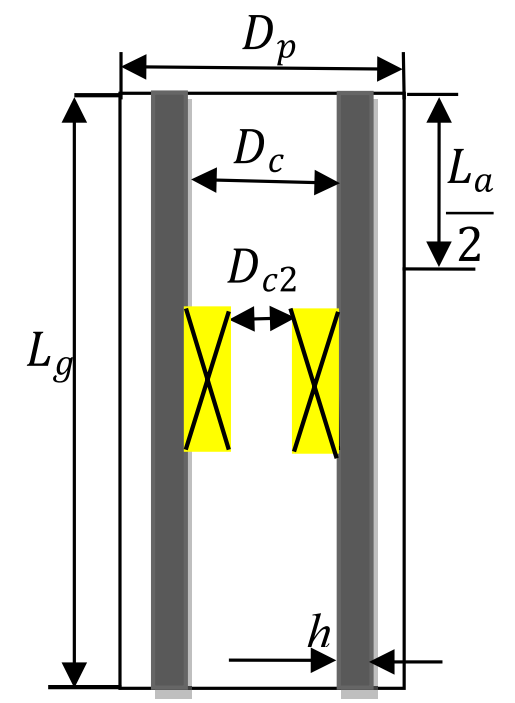

Fig. 1 Schematic of the magnetic circuit of the control valve

$$
\frac{D_{c}}{2}=L_{a}, \quad w_{c}=\sqrt{\frac{D_{c}^{2}}{2}-L_{a}^{2}}-L_{a} \quad h=\frac{D_{c}}{2}-w_{c}-\frac{D_{c 2}}{2}
$$

in which $w_{c}$ and $D_{c 2}$ are the width of coil and the distance between coils, respectively.

\section{MR fluid fabrication}

In this section, in order to fabricate the MR fluid, we used carbonyl iron $(\mathrm{Cl})$ powder with an average particle size less than $4 \mu \mathrm{m}$, density of $7.86 \mathrm{~kg} / \mathrm{cm}^{3}$ and $30 \mathrm{wt} \%$, produced by BASF $\mathrm{Co}$. $\mathrm{Cl}$ have been chosen due to a high magnetic saturation limit and magnetic permeability factor $[12,52]$. For investigating the sphericity value of $\mathrm{Cl}$ particles, the scanning electron micrograph (SEM) test is carried out and as shown in Fig. 2, the micron particles of carbonyl iron are in the desired spherical shapes.

There are some major reasons for choose of Polydimethylsiloxane oil (Silicone oil with viscosity 100 cst, density $0.0959 \mathrm{~g} / \mathrm{cm}^{3}$, KCC Co., Korea) as the base oil, i.e., the operation temperature domain is wide, the stability of $\mathrm{Cl}$ particle in this type of oil is high, and the magnetic permeability factor is low [53]. It is noteworthy to mention that a significant problem in MR dampers is stability [15]. To avoid this problem, three stabilizer materials are used in this work as below:

- Silicone dioxide nanopowder $\left(\mathrm{SiO}_{2}\right)$ : it has a high volume/ weight ratio does not increase the density of MR fluid. It the stabilizes the MR fluid by trapping the micronized carbonyl iron particles and lowering the surface tension of the fluid [54]. For this study, $1 \mathrm{wt} \%$ Silicone dioxide nanopowder $\left(\mathrm{SiO}_{2}\right)$ was selected. It was produced by

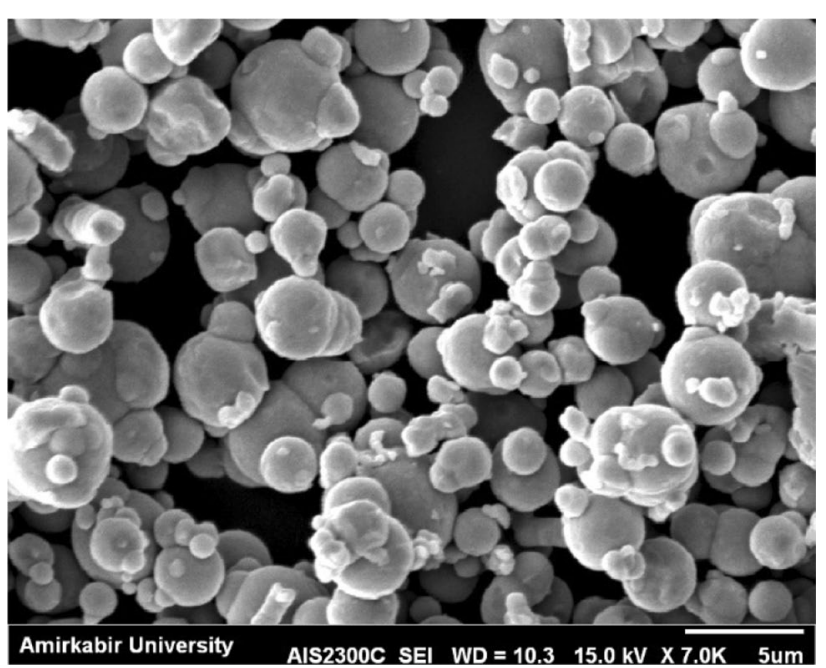

Fig. 2 The schematic of SEM of the CI particles 


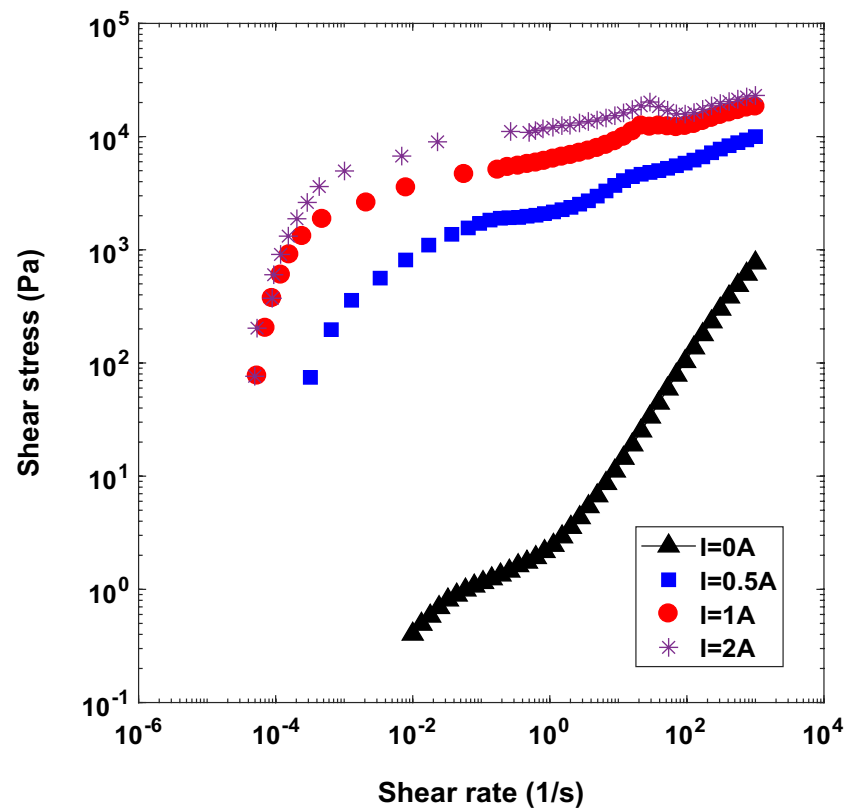

(a)

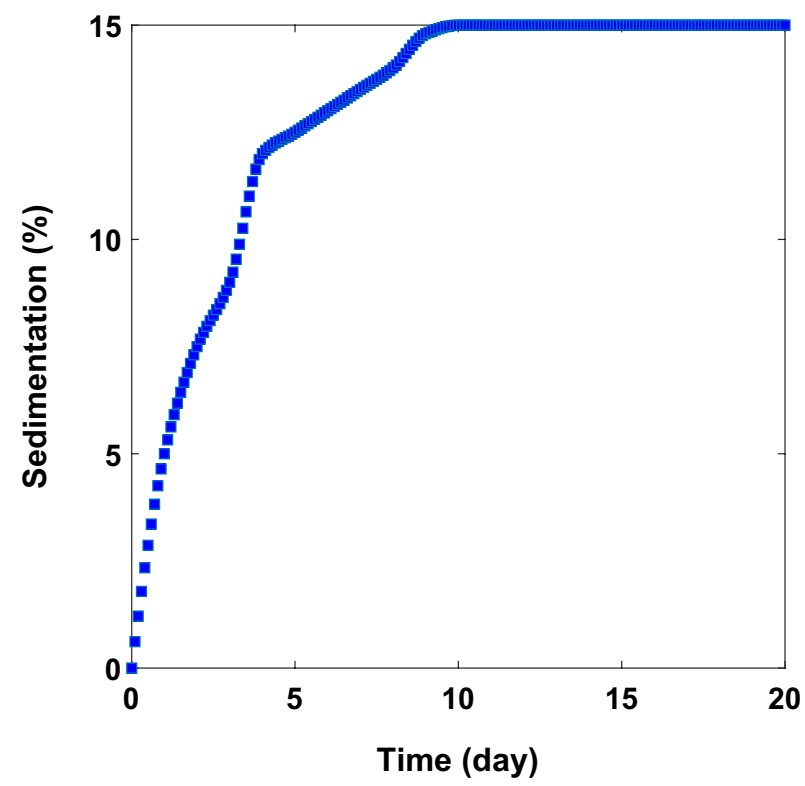

(c)

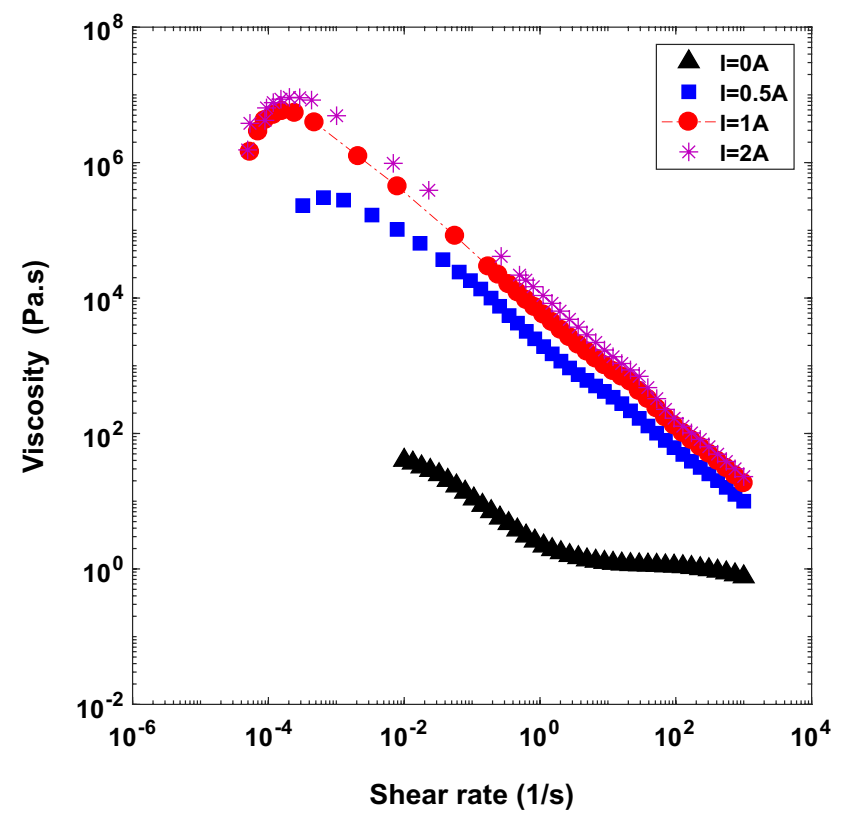

(b)

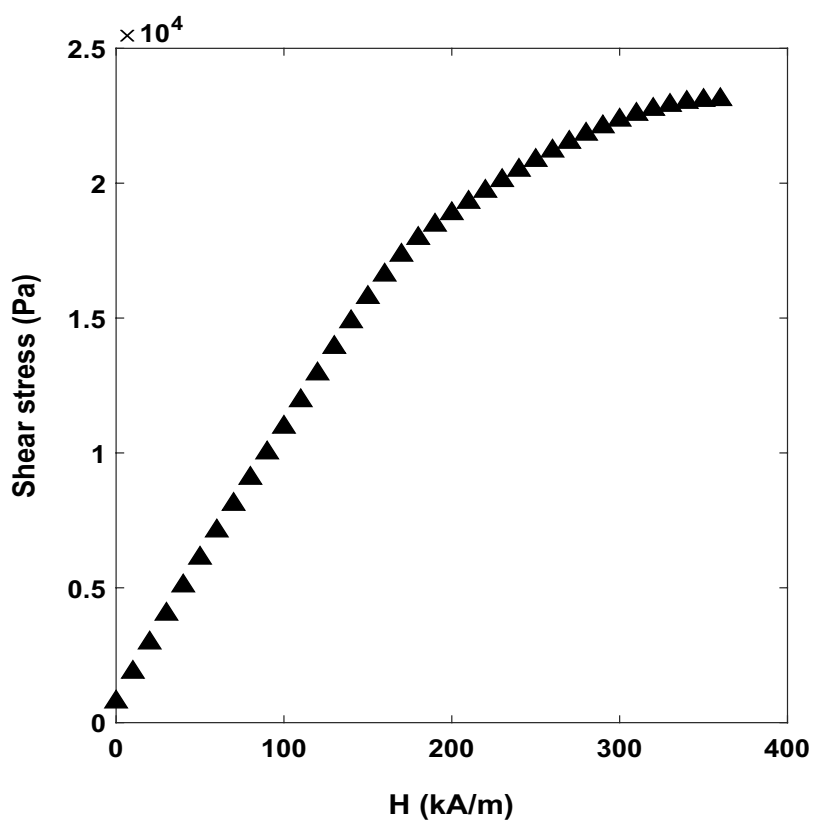

(d)

Fig. 3 The experimental results for the MR fluid. a The shear stress versus the strain rate of the MR fluid. $\mathbf{b}$ The viscosity versus the strain rate of the MR fluid. c The sedimentation of the MR fluid versus time. $\mathbf{d}$ The characteristic graph of the produced MR fluid

AEROSIL Co. with the density of $2.4 \mathrm{~g} / \mathrm{cm}^{3}$, and the particle size between 20 and $30 \mathrm{~nm}$, where the SCM test results showed a desired sphericity value.

- Stearic acid: with a density of $0.908 \mathrm{~g} / \mathrm{cm}^{3}, 1 \mathrm{wt} \%$, increases the stability of the MR fluid by rising the base fluid density and trapping the particles [19].

- Phosphoric acid: with a density of $1.885 \mathrm{~g} / \mathrm{cm}^{3}, 1 \mathrm{wt} \%$, stabilizes the MR fluid [55].
Mixing of MR fluid plays a significant role in the instability of MR fluid. In particular, initially, oil carrier, Silicone dioxide nanopowder, Phosphoric acid and Stearic acid are mixed in several steps, respectively, then by using FALCA magnetic mixer and ultrasonic mixer $(500 \mathrm{w})$, they are mixed for $30 \mathrm{~min}$. 


\subsection{The experimental results of the testing of MR fluid}

In this part, sedimentation and magneto-rheometry tests are carried out. As mentioned before, sedimentation of MR fluid in MR devices plays a crucial role in the performance of MR devices. After adding stabilizing materials to the MR fluid, sedimentation test is conducted. To this end, a little MR fluid in a glass measuring cylinder with total height "a" is considered, after a while, the height of the settled MR fluid is measured as " $b$ ". Furthermore, sedimentation rate of the MR fluid is calculated as $a / l \times 100$ [56]. The sedimentation rate of the MR fluid is calculated during 20 days as Fig. 3c. As depicted in Fig. 3c, the maximum sedimentation after 20 days is satisfying and is about $15 \%$. To identify the rheological properties of the MR fluid, the magneto-rheometry test (with MRC300 device) is performed. The variation of shear stress and viscosity of the MR fluid in different electrical currents are illustrated in Fig. 3a, b which reveals that the plastic viscosity is about $0.765 \mathrm{~Pa}$.s, yield shear stress is $22 \mathrm{kPa}$ and magnetic field intensity and maximum current are $300 \mathrm{kA} / \mathrm{m}$ and $2 \mathrm{~A}$, respectively. It is remarkable that the shear rate varies from 0.001 to $10001 / \mathrm{s}$ as well as the magneto-rheology test is done in four different currents of $0,0.5,1,2 \mathrm{~A}$. In order to eliminate the effect of hysteresis in the MR fluid, a new sample of the produced MR fluid is replaced in each electric current. In addition, the shear stress versus intensity of the magnetic field is shown in Fig. $3 \mathrm{~d}$. In the macroscopic view point, it could be inferred that the fabricated MR fluid has high shear stress which makes it a good candidate for use in MR damper.

In addition, to derive a relation between $\tau$ (in term of $\mathrm{Pa}$ ) and $H$ (in term of $\mathrm{kA} / \mathrm{m}$ ), considering the experimental data in Fig. 3d for BP model, we have:

$\tau=\left(765+105.52 H-0.003 H^{2}\right) \operatorname{sgn}(\dot{\gamma})+0.765 \dot{\gamma}$

\section{MR damper fabrication}

In the present section, the MR damper construction is described. It is a double tube type and works in the combination of shear and valve modes; i.e. it has two MR fluid reservoirs, inner and outer reservoir. Inner reservoir is the space between the inner cylinder and piston (or rod piston) and the outer reservoir is the space between the inner cylinder and outer tube. The MR damper includes the piston rod, control valve (including two magnetic core with both the coil and conventional pistons), the inner cylinder and the outer tube with soft ferromagnetic low carbon steel property, cap, rod guide, seal, MR fluid and stop bush. As mentioned previously, to have more uniform magnetic flux distributions, two coils are used. Also, the configuration of the control valve corresponds to the secondary flow path type. Finally, the configuration of the control valve and the MR damper are shown in Fig. $4 \mathrm{~b}$.

In addition, a magnetic analysis is conducted to illustrate the distribution of the magnetic flux in the control valve, employing ANSYS MAXWEL software. Two coils are wounded as series. Series circuit have a higher number of magnetic field lines compared to the parallel one, and there is a higher magnetic flux at the control valve edges. Although response time increases due to a higher induction factor and therefore a higher damping force is generated. In Fig. 5, the distribution and magnitude of the field density, field intensity and magnetic flux are

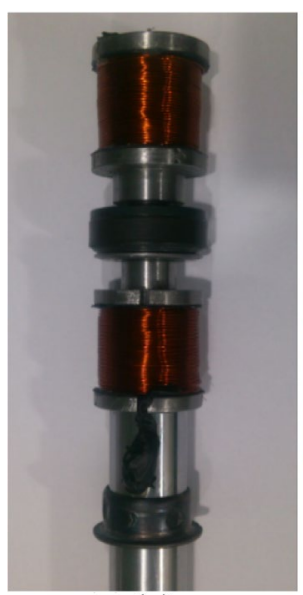

(a)

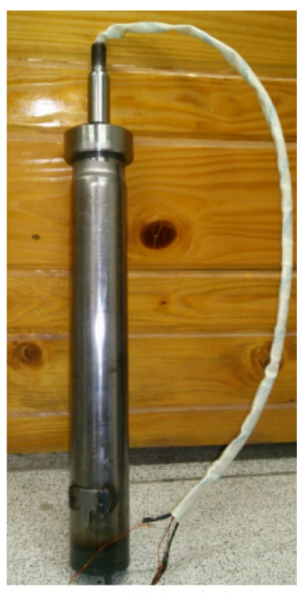

(b)
Fig. 4 The configuration of the $\mathbf{a}$ control valve and $\mathbf{b}$ the produced MR damper

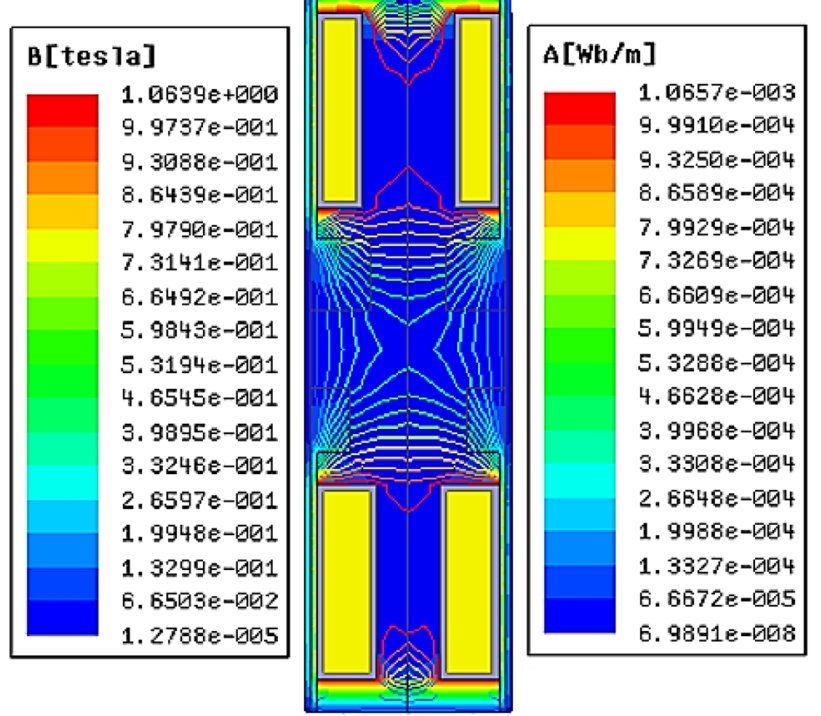

Fig. 5 Distribution of flux and magnetic field intensity at the series 
Table 1 Physical and geometrical properties of MR fluid and MR damper

\begin{tabular}{ll}
\hline$D_{\mathrm{p}^{\prime}} L_{\mathrm{a}^{\prime}}, h(\mathrm{~mm})$ & $32.0,8.0,1.0$ \\
$L_{\mathrm{g}^{\prime}} w_{\mathrm{c}^{\prime}} D_{\mathrm{c}}(\mathrm{mm})$ & $40.0,30.0,28.0$ \\
$d_{\mathrm{rod}}, D_{\mathrm{c} 2^{\prime}} d_{\mathrm{w}}(\mathrm{mm})$ & $20.0,16.0,0.7$ \\
$I_{\max }(\mathrm{A})$ & 2.0 \\
$N_{\mathrm{co}}(\mathrm{rev})$ & 300.0 \\
$H_{\mathrm{mr}}(\mathrm{kA} / \mathrm{m})$ & 300.0 \\
$\eta(\mathrm{Pa} . \mathrm{s})$ & 0.765 \\
$\tau_{0}(\mathrm{~Pa})$ & 765 \\
$\rho\left(\mathrm{g} / \mathrm{cm}^{3}\right)$ & 1.3 \\
\hline
\end{tabular}

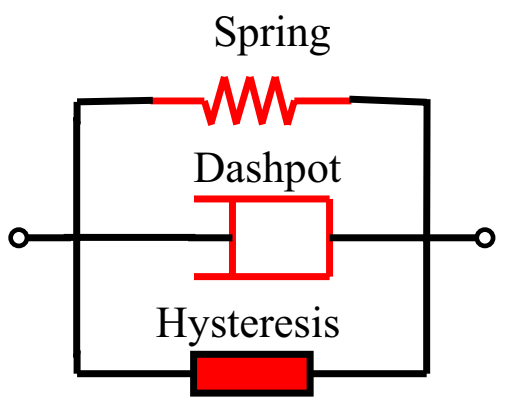

Fig. 6 Hysteresis model components

shown. It is noteworthy how choosing series or parallel winding depends upon the geometry of the control valve and the application of MR devices [57, 58].

Finally, the total material and geometrical parameters of the MR fluid and MR damper, are listed in Table 1.

\subsection{Testing of the MR damper}

In this section, the produced MR damper is tested to measure the damping force in each displacement and velocity. This work is carried out using a hydraulic shock-absorbing test machine (Lunik Spain) with a capacity of 1 ton, a maximum working frequency of $4.7 \mathrm{~Hz}$, a $300 \mathrm{~mm}$ course and frequency of $3.6 \mathrm{~Hz}$. This instrument displays the compression and tension force in correlation to a given displacement during the rod piston movement. The damping force is detected by a load cell mounted on the machine, which first receives it in the form of a voltage change (up to 10

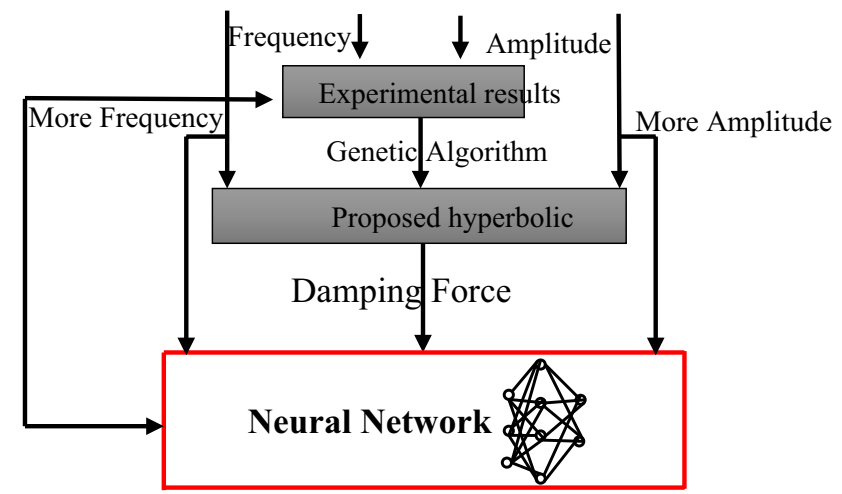

Fig. 7 Training of a neural network model for the MR damper

Volt) and then converts it to force magnitude in newton. The first trial is carried out at zero current, then the current is set as $0.5,1$ and $2 \mathrm{~A}$, respectively. The experimental results are presented in detail in the next section.

\section{Developing an analytical model of the MR damper}

\subsection{Hysteretic behavior of the MR dampers}

Several models have been proposed to illustrate the hysteresis behavior of the MR dampers. The Kwok model is used here to model the hysteresis characteristic of the MR dampers [59]. Figure 6 shows the schematic view of the hysteresis behavior of this model.

Mathematically, the model uses a hyperbolic tangent function to show the hysteresis response. It employs simple linear functions to represent the stiffness and viscosity in the damper. The model is defined as:

$f=c \dot{X}+k X+\alpha z+f_{0}$

$z=\tanh (\beta \dot{X}+\delta \operatorname{sign}(X))$

where $c, k, \alpha, z, f_{0}$ and $\delta$ are viscousity coefficient, stiffness coefficient, hysteresis factor, hysteresis variable, force offset and scale factor, respectively. GA is one of the most common optimization methods which due to its powerful nature can be used for identification of linear and nonlinear systems. On account of this characteristic, we
Table 2 The results of identification for the different input currents

\begin{tabular}{llllrll}
\hline Input current $(\mathrm{A})$ & \multicolumn{1}{c}{$c$} & \multicolumn{1}{c}{$k$} & $\alpha$ & $f_{0}$ & $\beta$ & $\delta$ \\
\hline$I=0$ & 29.013 & 187.396 & 6.993 & -0.971 & 41.884 & 9.887 \\
$I=0.5$ & 115.705 & 574.552 & 65.925 & 2.863 & 45.538 & 8.307 \\
$I=1$ & 166.793 & 1719.929 & 89.812 & -1.948 & 18.092 & 4.16 \\
$I=2$ & 339.705 & 2892.696 & 98.974 & 1.15 & 43.898 & 9.542 \\
\hline
\end{tabular}




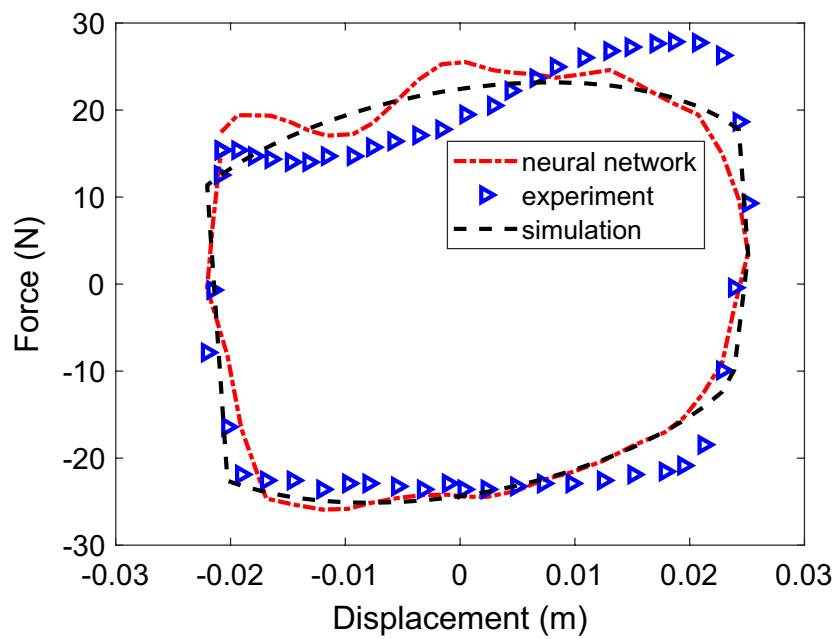

(I=0A)

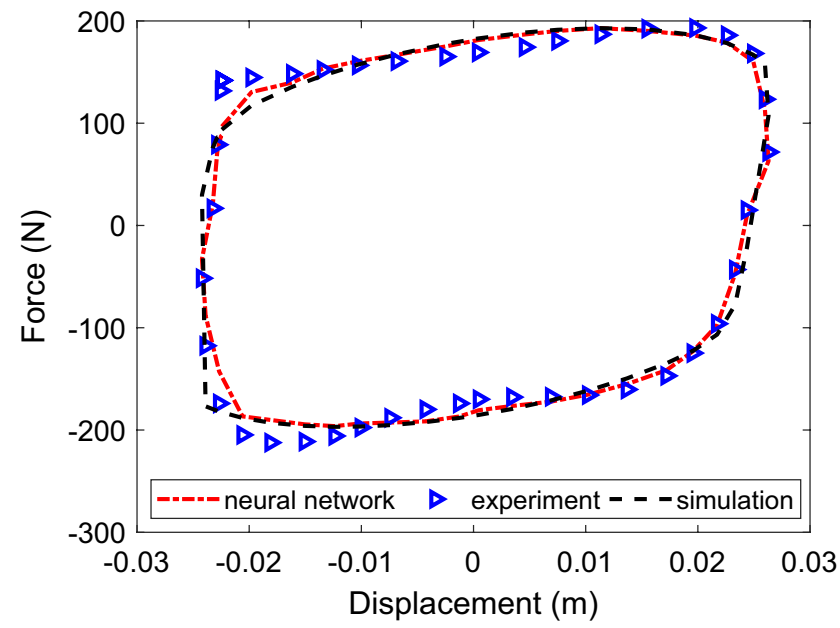

$(\mathrm{I}=\mathbf{1 A})$

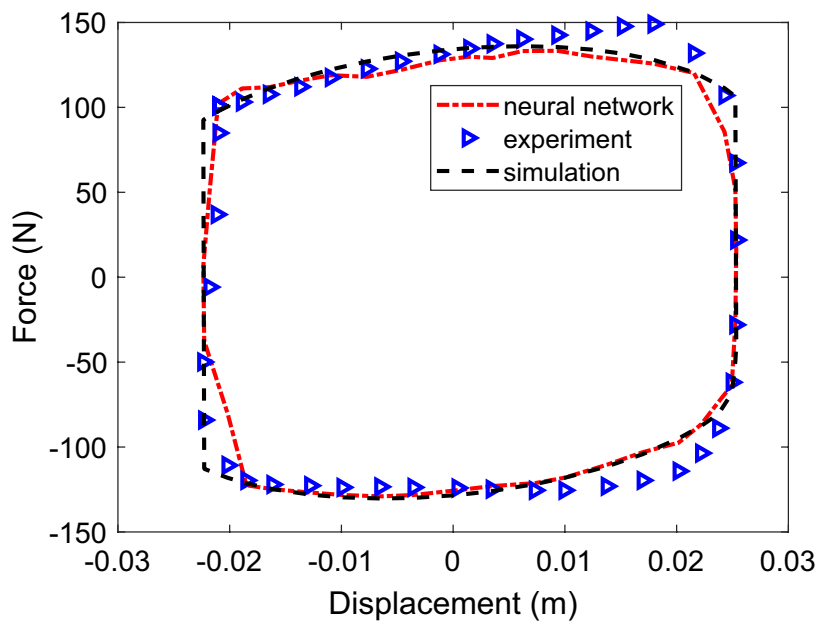

$(\mathrm{I}=\mathbf{0 . 5 A})$

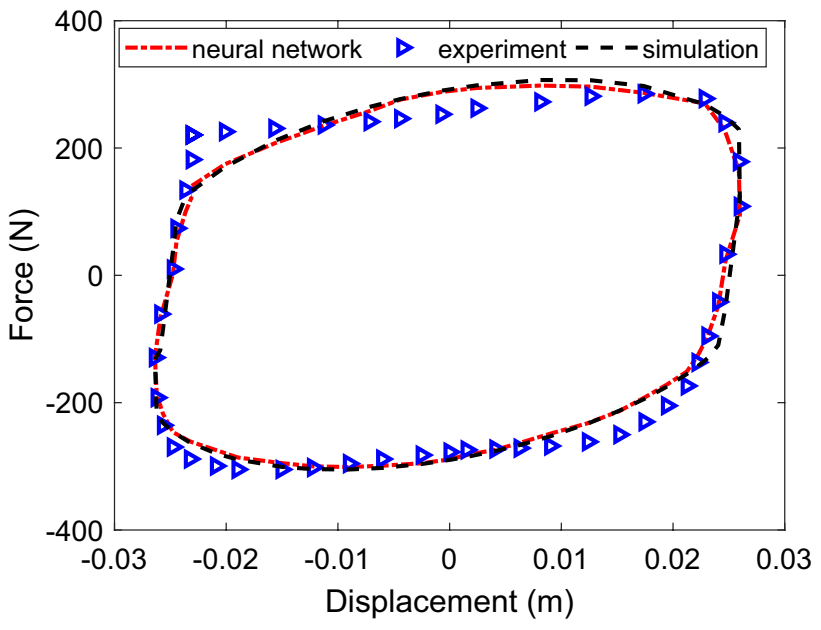

$(\mathrm{I}=\mathbf{2 A})$

Fig. 8 Comparison of the damping force versus displacement between the experimental data, hysteresis model and the neural network prediction in four different currents $0,0.5,1$ and $2 \mathrm{~A}$

can use GA for parameter identification of the presented MR damper model. Parameter identification, using the GA is based on calculating the appropriate fitness function. The function compares the output data from the experiment and simulation model. Minimization of this function gives more accurate parameters [60]. The main advantage of GA is its relatively good precision in identifying the parameters in presence of noise and quantized data. On the other hand, the system complexity together with the numerous number of parameters makes the procedure, time consuming. As indicated in Eqs. (4) and (5), the model has six undefined parameters, i.e., $c, k, \alpha, f_{0}, \beta$ and $\delta$. All the parameters except the initial displacement $X$ and velocity $\dot{X}$ are set as inputs. The fitness function used in the GA is derived from the difference between the experimental and simulation results. Table 2 reports the results of the identification for different input currents to the damper.

fitness $=\frac{1}{n} \sum_{i=1}^{n}\left|F_{d, i}^{\exp }-F_{d, i}^{s i m}\right|$

\subsection{Neural network model construction}

Several researchers proposed parameter-based models. However, parametric methods involve some assumptions for the model. Also the parametric models cannot predict the behavior of the system in different currents fed to the damper, so in this case, the use of numerical predictions in proposing a universal model 


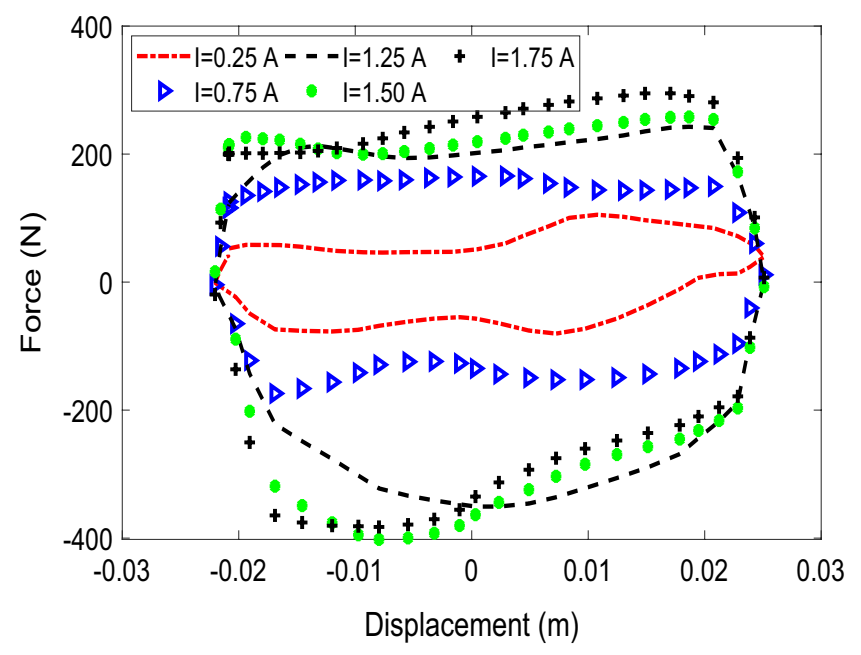

(a)

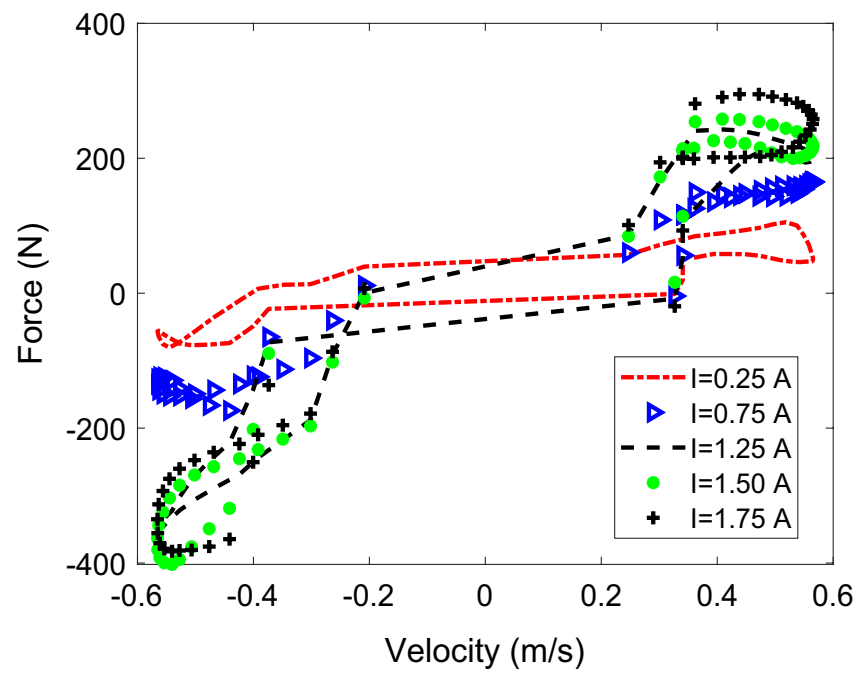

(b)

Fig. 9 Comparative predicted results of neural network in other currents. a Damping force versus displacement, $\mathbf{b}$ damping force versus velocity

is inevitable. Furthermore, non-parametric models are robust and applicable to linear, non-linear and hysteresis systems [61]. For the MR damper modeling, Chang and Roschke [62] proposed a non-parametric model using the neural networks. In this study, a feedforward network is employed and the network is trained on all the input and output information of the MR dampers. The data required for the training is derived from both the experiment and simulation model generated in Sect. 6 . Figure 7 shows the schematic view of training of the neural network for the MR damper.

\section{Results and discussion}

In this section, experimental results, the results of the hysteresis model and the neural network outcome are presented. The experiments were carried out in four currents $0,0.5,1,2 \mathrm{~A}$. The results for three cases are shown in Fig. 8.

It is well known that at higher currents (or magnetic intensity), the amount of the damping force rises and the figure of the damping force versus displacement is stretched. The main reason for this physical phenomenon is that by increasing the magnetic field, the magnetic particles in MR fluid are aligned so that the viscosity of the MR fluid increases; consequently, the damping force is enhanced. As mentioned in the previous section, despite the hysteresis model, to predict the dynamic characteristic of the MR damper in other currents and frequencies, the neural network is implemented. The results of this modeling are presented in Fig. 9.

\section{Summary and conclusion}

In the present work, a novel MR fluid with different components and additives was produced. In the present MR fluid in order to lower the sedimentation and agglomeration of the particles, Phosphoric acid, Stearic acid as well as Silicone dioxide nanopowder were utilized. Then, rheometrical characteristics of the MR fluid under rheometry test was calculated. Afterward, the conceptual design of the MR damper was performed. The MR damper was fabricated Based on the geometrical design, magnetic analysis and rheological properties of MR fluid. Also, the dynamic test was carried out to identify the dynamic mechanical parameters. A hysteresis model based on the experimental data was applied to predict the dynamic behavior of the MR damper. Employing a neuro network algorithm, the dynamic response of the MR damper were identified under different conditions (e.g., frequency, amplitude of the displacement and, electrical current). The predictions of the proposed model and the neural network, are in good agreement with the experimental data. Also, the neural network has a broad potential in predestining of the MR damper in other currents. 


\section{Compliance with ethical standards}

Conflict of interest The authors declare that they have no competing interests.

\section{References}

1. Smith RC (2005) Smart material systems: model development, vol 32. Siam, North Carolina

2. Banks $\mathrm{H}$, Smith RC (2000) Hysteresis modeling in smart material systems. Appl Mech Eng 5(1):31-45

3. Baghani M, Dolatabadi R, Baniassadi M (2017) Developing a finite element beam theory for nanocomposite shapememory polymers with application to sustained release of drugs. Sci Iran 24(1):249-259

4. Perales-Martínez IA et al (2017) Enhancement of a magnetorheological PDMS elastomer with carbonyl iron particles. Polym Testing 57:78-86

5. Kumar A, Mangal S (2012) Properties and applications of controllable fluids: a review. Int J Mech Eng Res 2(1):57-66

6. Yarali E, Baniassadi M, Baghani M (2019) Numerical homogenization of coiled carbon nanotube reinforced shape memory polymer nanocomposites. Smart Mater Struct 28(3):035026

7. Spencer B Jr et al (1997) Phenomenological model for magnetorheological dampers. J Eng Mech 123(3):230-238

8. Poursafar J, Bashirpour M, Kolahdouz M, Vakilipour Takaloo A, Masnadi-Shirazi M, Asl-Soleimani E (2018) Ultrathin solar cells with Ag meta-material nanostructure for light absorption enhancement. Sol Energy 166:98-102

9. Carlson JD, Catanzarite D, St. Clair K (1996) Commercial magneto-rheological fluid devices. Int J Mod Phys B 10(23-24):2857-2865

10. Dyke $S$ et al (1996) Modeling and control of magnetorheological dampers for seismic response reduction. Smart Mater Struct 5(5):565

11. Rabinow J (1948) The magnetic fluid clutch. Electr Eng 67(12): 1167

12. Ghorbani S, Bashirpour M, Kolahdouz M, Neshat M, Mansouree M, Forouzmehr M (2014) Improving efficiency of THz photoconductive antennas using nano plasmonic structure. In: Optics InfoBase Conference Papers

13. Ahamed R, Ferdaus MM, Li Y (2016) Advancement in energy harvesting magneto-rheological fluid damper: a review. KoreaAustralia Rheol J 28(4):355-379

14. Ha SH, Seong M-S, Choi S-B (2013) Design and vibration control of military vehicle suspension system using magnetorheological damper and disc spring. Smart Mater Struct 22(6):065006

15. Yang Y, Li L, Chen G (2009) Static yield stress of ferrofluid-based magnetorheological fluids. Rheol Acta 48(4):457-466

16. Vékás $L$ (Year) Ferrofluids and magnetorheological fluids. In: Advances in science and technology of conference. Trans Tech Publ

17. Wang X, Gordaninejad F (2006) Study of magnetorheological fluids at high shear rates. Rheol Acta 45(6):899-908

18. Yoo J-H, Wereley NM (2002) Design of a high-efficiency magnetorheological valve. J Intell Mater Syst Struct 13(10):679-685

19. Ashtiani M, Hashemabadi S, Ghaffari A (2015) A review on the magnetorheological fluid preparation and stabilization. J Magn Magn Mater 374:716-730

20. Pan J et al (2019) Research on material removal model and processing parameters of cluster magnetorheological finishing with dynamic magnetic fields. Int J Adv Manuf Technol 100(9):2283-2297

21. Jinaga $R$ et al (2019) Design, fabrication and testing of a magnetorheologic fluid braking system for machine tool application. SN Appl Sci 1(4):328

22. Hu G et al (2019) Damping performance analysis of magnetorheological damper with serial-type flow channels. Adv Mech Eng 11(1):1687814018816842

23. Li J et al (2018) Electromechanical characteristics and numerical simulation of a new smaller magnetorheological fluid damper. Mech Res Commun 92:81-86

24. Nguyen Q-H, Choi S-B (2009) Optimal design of MR shock absorber and application to vehicle suspension. Smart Mater Struct 18(3):035012

25. Lee H-G et al (2011) Performance evaluation of a quarter-vehicle MR suspension system with different tire pressure. Int J Precis Eng Manuf 12(2):203-210

26. Zhu X, Lai C (2012) Design and performance analysis of a magnetorheological fluid damper for drillstring. Int J Appl Electromagnet Mech 40(1):67-83

27. Zhu S et al (2016) A novel design of magnetorheological damper with annular radial channel. Shock Vib

28. Wang D, Liao WH (2011) Magnetorheological fluid dampers: a review of parametric modelling. Smart Mater Struct 20(2):023001

29. Wereley NM, Pang $L$ (1998) Nondimensional analysis of semiactive electrorheological and magnetorheological dampers using approximate parallel plate models. Smart Mater Struct 7(5):732

30. Yu J, Dong X, Wang W (2016) Prototype and test of a novel rotary magnetorheological damper based on helical flow. Smart Mater Struct 25(2):025006

31. Adibi H, Yarali E, RamezanShams A (2017) Design, fabricate and testing the novel Magnetorheologic damper involving stabilizer nanoparticles of silicone. Modares Mech Eng 17(8):252-258

32. Zhao Y-L, Xu Z-D (2018) A hysteretic model considering Stribeck effect for small-scale magnetorheological damper. Smart Mater Struct 27(6):065021

33. Xinchun $G$ et al (2015) A novel self-powered MR damper: theoretical and experimental analysis. Smart Mater Struct 24(10):105033

34. Guan $X$, Ru Y, Huang $Y$ (2017) A novel velocity self-sensing magnetorheological damper: design, fabricate, and experimental analysis. J Intell Mater Syst Struct 30(4):497-505

35. Chooi WW, Oyadiji SO (2008) Design, modelling and testing of magnetorheological (MR) dampers using analytical flow solutions. Comput Struct 86(3):473-482

36. Liu J et al (2018) Realizing negative Poisson's ratio in unstressed spring networks. arXiv preprint arXiv: 1810.10768

37. Lee T-H, Choi S-B (2018) On the response time of a new permanent magnet based magnetorheological damper: experimental investigation. Smart Mater Struct 28(1):014001

38. Choi Y-T, Wereley NM (2002) Comparative analysis of the time response of electrorheological and magnetorheological dampers using nondimensional parameters. J Intell Mater Syst Struct 13(7-8):443-451

39. Milecki A (2002) Investigation of dynamic properties and control method influences on MR fluid dampers' performance. J Intell Mater Syst Struct 13(7-8):453-458

40. Koo J-H, Goncalves FD, Ahmadian M (2006) A comprehensive analysis of the response time of MR dampers. Smart Mater Struct 15(2):351

41. Bhatnagar R (2018) A nonlinear transient model for magnetorheological damper response for a time varying field controllable yield shear stress. J Braz Soc Mech Sci Eng 40(9):425 
42. Peng Y, Yang J, Li J (2018) Parameter identification of modified Bouc-Wen model and analysis of size effect of magnetorheological dampers. J Intell Mater Syst Struct 29(7):1464-1480

43. Bharathi Priya C, Gopalakrishnan N (2019) Temperature dependent modelling of magnetorheological (MR) dampers using support vector regression. Smart Mater Struct 28(2):025021

44. Choi SB, Han YM (2005) Hysteretic behavior of a magnetorheological fluid: experimental identification. Acta Mech 180(1):37-47

45. Carlson JD, Jolly MR (2000) MR fluid, foam and elastomer devices. Mechatronics 10(4-5):555-569

46. Chaudhuri A et al (2006) Rheological parameter estimation for a ferrous nanoparticle-based magnetorheological fluid using genetic algorithms. J Intell Mater Syst Struct 17(3):261-269

47. Ding $Y$ et al (2014) Simplified design method for shearvalve magnetorheological dampers. Earthq Eng Eng Vib 13(4):637-652

48. Cheng DK (1989) Field and wave electromagnetics. AddisonWesley, Boston

49. Ferdaus MM et al (2014) Optimal design of magneto-rheological damper comparing different configurations by finite element analysis. J Mech Sci Technol 28(9):3667-3677

50. Gołdasz J, Sapiński B (2015) Insight into magnetorheological shock absorbers. Springer, New York

51. Golinelli N, Spaggiari A (2015) Design of a novel magnetorheological damper with internal pressure control. Frattura ed Integritá Strutturale 9(32):13-23

52. Kciuk S, Turczyn R, Kciuk M (2010) Experimental and numerical studies of MR damper with prototype magnetorheological fluid. J Achiev Mater Manuf Eng 39(1):52-59

53. Kciuk M, Kciuk S, Turczyn R (2009) Magnetorheological characterisation of carbonyl iron based suspension. J Achiev Mater Manuf Eng 33(2):135-141

54. Ashtiani M, Hashemabadi SH (2015) The effect of nano-silica and nano-magnetite on the magnetorheological fluid stabilization and magnetorheological effect. J Intell Mater Syst Struct 24(14):1887-1892

55. Świtoński E et al (2007) Prototype magnetorheological fluid damper for active vibration control system. J Achiev Mater Manuf Eng 21(1):55-62

56. Tu J et al (2011) Design and fabrication of 500-kN large-scale MR damper. J Intell Mater Syst Struct 22(5):475-487

57. Zheng J et al (2014) Magnetic circuit design and multiphysics analysis of a novel MR damper for applications under high velocity. Adv Mech Eng 6:402501

58. Kelso SP (Year) Experimental characterization of commercially practical magnetorheological fluid damper technology. In: SPIE's 8th annual international symposium on smart structures and materials of conference. International Society for Optics and Photonics

59. Kwok N et al (2006) A novel hysteretic model for magnetorheological fluid dampers and parameter identification using particle swarm optimization. Sens Actuators A 132(2):441-451

60. Man K-F, Tang K-S, Kwong S (1996) Genetic algorithms: concepts and applications [in engineering design]. IEEE Trans Ind Electron 43(5):519-534

61. Ehrgott R, Masri S (1992) Modeling the oscillatory dynamic behaviour of electrorheological materials in shear. Smart Mater Struct 1(4):275

62. Chang C-C, Roschke P (1998) Neural network modeling of a magnetorheological damper. J Intell Mater Syst Struct 9(9):755-764

Publisher's Note Springer Nature remains neutral with regard to jurisdictional claims in published maps and institutional affiliations. 